\title{
Atomic Carbon Chains-Mediated Carriers Transfer over Polymeric Carbon Nitride for Efficient Photocatalysis
}

Yunxiang $\mathrm{Li}^{\mathrm{a}, \mathrm{b}}$, Jian Ren ${ }^{\mathrm{e}, \mathrm{f}}$, Shuxin Ouyang ${ }^{\mathrm{c}, \mathrm{d}}$, Weishu Hou ${ }^{\mathrm{c}, \mathrm{d}}$, Tristan Petit ${ }^{\mathrm{e}}$, Hui Song $^{\mathrm{a}, \mathrm{b}}$, Huayu Chen ${ }^{\mathrm{c}, \mathrm{d}}$, Davin Phillo ${ }^{\mathrm{a}, \mathrm{b}}$, Tetsuya Kako ${ }^{\mathrm{b}}$ and Jinhua Ye $\mathrm{e}^{\mathrm{a}, \mathrm{b}, \mathrm{c}, \mathrm{d},{ }^{*}}$

${ }^{a}$ Graduate School of Chemical Science and Engineering, Hokkaido University, Sapporo 060-0814, Japan

binternational Center for Materials Nanoarchitectonics (WPI-MANA), National Institute for Materials Science (NIMS), 1-1 Namiki, Tsukuba 305-0047, Japan

${ }^{\mathrm{c}}$ TJU-NIMS International Collaboration Laboratory, School of Materials Science and Engineering, Tianjin University, Tianjin 300072, P. R. China

${ }^{\mathrm{d} C}$ Collaborative Innovation Center of Chemical Science and Engineering (Tianjin), Tianjin 300072, P. R. China

${ }^{\mathrm{e}}$ Methods for Material Development, Helmholtz-Zentrum Berlin für Materialien und Energie GmbH (HZB), Albert-Einstein-Strasse 15, Berlin, 12489, Germany

fDepartment of Physics, Freie Universität Berlin, Arnimallee 14, Berlin, 14195, Germany

E-mail: Jinhua.YE@nims.go.jp 


\begin{abstract}
Atomic structure tailoring towards high electron mobility is an essential approach to maximizing the solar energy-conversion efficiency of polymeric carbon nitride $(\mathrm{CN})$ but still presents a significant challenge. Here we construct a smooth carrier channel in the basal plane of $\mathrm{CN}$ by filling the rich defects in layer with various kinds of short carbon chains, whereby the light-induced carriers transfer kinetics is boosted distinctly. Consequently, the optimal carbon chains-planted $\mathrm{CN}$ delivers a remarkably enhanced photocatalytic performance, achieving a 13.2 and 29.2-fold improvement in $\mathrm{H}_{2}$ evolution and $\mathrm{CO}_{2}$ reduction, respectively. This study provides an in-depth insight into the modulation of in-plane electrical conductivity at molecular scale over $\mathrm{CN}$ and offers new opportunities for reinforcing the reaction kinetics of organic-based photocatalysts.
\end{abstract}

\title{
Keywords:
}

Carbon nitride; Conductivity engineering; Carbon chains; Photocatalytic $\mathrm{H}_{2}$ generation; $\mathrm{CO}_{2}$ reduction

\section{Introduction}

The generation of clean and renewable chemicals from $\mathrm{H}_{2} \mathrm{O}$ and $\mathrm{CO}_{2}$ using photocatalysts is a highly promising solution to the conversion and storage of solar energy. ${ }^{[1]}$ The development of efficient, durable, and sustainable photocatalysts is always at the heart of photocatalytic research. Layered polymeric carbon nitride $(\mathrm{CN})$, a metal-free and chemically stable material, has attracted intense interest in all kinds of solar to chemical energy conversion process including $\mathrm{H}_{2} / \mathrm{O}_{2}$ evolution from water splitting and $\mathrm{CO}_{2}$ reduction. ${ }^{[2-10]}$ Owing to the unique chemical composition and layered feature, polymeric $\mathrm{CN}$ possesses high adjustability and compatibility and thus offer us huge potential to targetedly tailor its structure and properties towards diverse applications. ${ }^{[11-19]}$

However, similar to other kinds of organic framework, e.g., metal-organic frameworks (MOFs), and covalent organic frameworks (COFs), efficient solar-toenergy conversion over polymeric $\mathrm{CN}$ still encounters great challenges of sluggish 
radiative carriers transfer within layers due to the long transportation distance and rich defects in layers. Notably, these defects in polymeric $\mathrm{CN}$ are mainly related to $\mathrm{NH}_{2}$ and $\mathrm{NH}$ groups because of the incomplete polymerization of amide groups-rich precursors. ${ }^{[20]}$ Abundant intralayered hydrogen bonds are thus (as illustrated by the dash line in Scheme 1a) formed in the defect regions. ${ }^{[20,21]}$ As a result, the transport of charge carriers across the layers of $\mathrm{CN}$ is signally different from that of other materials only containing covalent bonds within the layers, e.g., $\mathrm{MoS}_{2}$ with Mo-S bonds and nitrogen doped graphene with C-N/C-C bonds. ${ }^{[20]}$ These hydrogen bonds are not favorable relative to covalent bonds for the transport of photocarriers within the plane and thus cause the high localization of photocarriers within the tri-s-triazine units. ${ }^{[20,22]}$

The direct atomic engineering of intralayered defects/motifs is the most fundamental and straightforward pathway to facilitate the transport of carriers in the layered $\mathrm{CN}$ and also can provide deeper insight into the behavior of the carriers in CN. Recently, several pioneering approaches have been sought in the perspective of this sight. ${ }^{[12,23-25]}$ First, Liu et al. selectively broke the hydrogen bond via reducing the residual $\mathrm{NH}_{2} / \mathrm{NH}$ groups at high temperature, in part resulting in a remarkable suppression of radiative carriers recombination in photocatalyst. ${ }^{[20]}$ Another reported strategy is to implant the carbon ring including two similar types - " $\mathrm{C}_{\text {ring" }}$ and "gradually doped graphited carbon rings" into the planar layer of $\mathrm{CN}{ }^{[23,24]} \mathrm{In}$ the case of $\mathrm{C}_{\text {ring }}$ modified $\mathrm{CN}$, the in-plane inserted $\mathrm{C}_{\text {ring }}$ not only expedites the carriers separation but also promotes the photoelectron transport. ${ }^{[23]}$ Similarly, carbon quantum dots, with relatively larger size compared to atomic carbon ring, were also implanted in the 2D plane of $\mathrm{CN}$ seamlessly to facilitate the photoelectron transport. ${ }^{[25]}$ However, these strategies above-mentioned still suffer from several drawbacks, such as moderate photocatalytic performance or complicated and energy consumption procedures. Besides, precise and targeted basal plane engineering of $\mathrm{CN}$ with atomic carbon species smaller than aforementioned carbonbased dopants, i.e., short carbon chains, has not been intentionally attempted yet and still remains a significant challenge. 
Herein, we propose a conceptual and facile strategy to construct carbon chains incorporated $\mathrm{CN}$, in which various kinds of carbon chains connected the in-plane defects of $\mathrm{CN}$ via strong covalent $\mathrm{C}-\mathrm{N}$ bonds. Notably, these mediated carbon chains serve as the electron transport channel to reinforce the separation and transfer of photogenerated carriers in the basal plane of $\mathrm{CN}$. Therefore, the optimized sample delivers a remarkably enhanced photocatalytic performance by achieving a 13.2 and 29.2-fold improvement for water reduction and $\mathrm{CO}_{2}$ reduction, respectively, as well as good stability without noticeable attenuation.

\section{Experimental}

\subsection{Materials preparation}

All reagents were analytical grade and used without further purification. The deionized water (18.2 M $\Omega$ ) that was used throughout all experiments was produced using a Millipore Direct-Q System. The pristine polymeric CN (denoted as CN) was fabricated by annealing $1.5 \mathrm{~g}$ melamine up to $550{ }^{\circ} \mathrm{C}$ at a rate of $2.3{ }^{\circ} \mathrm{C} \min ^{-1}$ and kept for $4 \mathrm{~h}$ in a covered $\mathrm{Al}_{2} \mathrm{O}_{3}$ crucible. After cooled down to room temperature, the product was grounded to obtain a powder sample. To fabricate the carbon chains linked $\mathrm{CN}$, melamine and various linkers including glycine, $\beta$-Alanine, 4-Aminobutyric acid, Lcysteine, Allyl thiourea and L-Arginine were first mixed fully. Using the linker glycine as an example, $1.5 \mathrm{~g}$ melamine and certain amount of glycine $(5,10,15,20,30 \mathrm{mg})$ were first dissolved in $20 \mathrm{~mL}$ deionized water with stirring and heated at $\sim 80{ }^{\circ} \mathrm{C}$ to evaporate the water. Then, the white mixture followed the identical calcination procedure of $\mathrm{CN}$ as mentioned above. The samples thus obtained were named as $\mathrm{CN}$ xGly, where $x(\mathrm{mg})$ represents the amount of glycine. As shown in Fig. 3a,b, the optimized amount of glycine was $10 \mathrm{mg}$. Therefore, for other linkers, the amount was roughly fixed at $10 \mathrm{mg}$ to evaluate the validity of different linkers on photocatalytic activity. The CN-10Gly-(two steps) was synthesis by heating the mixture of $\mathrm{CN}$ ( 600 $\mathrm{mg}$, derived from $1.5 \mathrm{~g}$ melamine) and $10 \mathrm{mg}$ glycine at $550{ }^{\circ} \mathrm{C}$.

\subsection{Physicochemical characterization}


XRD patterns of the as-prepared samples were collected with a powder X-ray diffractometer $(\mathrm{Cu} \mathrm{K} \alpha$ radiation source, X'pert powder, PANalytical B.V., Netherlands). UV-visible (UV-vis) diffuse reflectance spectra of the samples were recorded on an UV-visible spectrophotometer (UV-2700, Shimadzu, Japan) equipped with an integrating sphere, then converted into absorption spectra by Kubelka-Munk transformation. Nitrogen adsorption-desorption isotherms, and surface area were recorded using a BEL SORP-mini II (BEL Japan INC., Japan), and were calculated via the Brunauer-Emmett-Teller (BET) method. Infrared transmission spectra were collected using a Fourier Transform infrared (FT-IR) spectrophotometer (Nicolet 4700, Thermo SCIENTIFIC, America). The in situ FT-IR measurements were conducted on a JASCO FTIR-6300 spectrometer under argon with a homemade chamber. The Photoluminescence (PL) properties were investigated using a fluorescence spectrophotometer (Fluorolog-3, HORIBA Scientific, America). X-ray photoelectron spectroscopy (XPS) measurements were carried out on XPS instrument (Escalab 250, Thermo SCIENTIFIC, America). All binding energies were referenced to the C $1 s$ peaks of the surface adventitious carbon at $284.8 \mathrm{eV}$. The morphologies of samples were characterized with Field Emission Scanning Electron Microscope (JSM-6701F, JEOL Co., Japan) and Transmission Electron Microscope (TEM) (JEM-2100F, JEOL Co., Japan). X-ray absorption spectroscopy measurements were carried on CN samples drop casted on conductive Si wafer. The data were collected at the U49/2 PGM1 undulator beamline of the BESSY II synchrotron radiation source using the LiXEdrom endstation. The XAS were measured in the total electron yield mode by scanning the samples in the energy range of $276 \sim 310 \mathrm{eV}$ (for C K-edge) or $395 \sim 420 \mathrm{eV}$ (for N Kedge) in $0.1 \mathrm{eV}$ steps. The XAS spectra in the figures have been normalized to the background before and after the main features.

\subsection{Photocatalytic $\mathrm{H}_{2}$ evolution test}

The photocatalytic $\mathrm{H}_{2}$ evolution test was carried out in a glass reactor with a closed gas circulation system. The light source was a $300 \mathrm{~W}$ Xe lamp. $50 \mathrm{mg}$ photocatalyst was firstly dispersed with a magnetic stirrer in triethanolamine aqueous solution (30 
$\mathrm{mL}$ of triethanolamine and $220 \mathrm{~mL}$ of $\mathrm{H}_{2} \mathrm{O}$ ) and was loaded with $2 \mathrm{wt} \% \mathrm{Pt}$ as the cocatalyst via a photodeposition process (under UV-visible light irradiation, $\lambda>350 \mathrm{~nm}$ ). After Pt loading and vacuumizing, $\mathrm{H}_{2}$ evolution over the photocatalyst was carried out under visible light irradiation (with an L42 filter, $\lambda>400 \mathrm{~nm}$ ). The evolved $\mathrm{H}_{2}$ was analyzed by online gas chromatography (GC-8A; Shimadzu Corp., Japan) equipped with a thermal conductivity detector.

\subsection{Photocatalytic $\mathrm{CO}_{2}$ reduction test}

The photocatalytic $\mathrm{CO}_{2}$ evolution test was carried out in a glass reactor with a closed gas circulation system. $20 \mathrm{mg}$ photocatalyst, $1 \mu \mathrm{mol} \mathrm{CoCl}_{2}$, and $15 \mathrm{mg}$ 2, 2-bipyridine were dispersed with a magnetic stirrer in solution $\left(50 \mathrm{~mL}\right.$ acetonitrile, $10 \mathrm{~mL} \mathrm{of} \mathrm{H}_{2} \mathrm{O}$ and $2 \mathrm{~mL}$ of triethanolamine). After complete vacuumizing, the reaction system was injected with pure $\mathrm{CO}_{2}(\sim 80 \mathrm{kPa})$. The produced gases were analyzed by a gas chromatography. The light source was a $300 \mathrm{~W}$ Xe lamp (with a L42 cutoff filter and water filter, $\lambda>400 \mathrm{~nm}$ ). The water filter used here is to remove the IR irradiation.

\subsection{Photoelectrochemical measurement}

The photoelectrodes were prepared according to previously reported method. The indium doped tin oxide (ITO) substrates were cleaned by ultrasonication in distilled water, absolute ethanol, and isopropanol for $15 \mathrm{~min}$ sequentially, and then dried in vacuum. $5 \mathrm{mg}$ of photocatalysts and $20 \mu \mathrm{L}$ of Nafion solution (5 wt $\%$ ) were dispersed in $1 \mathrm{~mL}$ ethanol by at least $30 \mathrm{~min}$ sonication to prepare a homogeneous catalyst colloid. Then, $100 \mu \mathrm{L}$ of the catalyst colloid was deposited onto areas of $\approx 1 \mathrm{~cm}^{2}$ of the ITO conductive glass and then was dried in air to form the working electrode. The photoelectrochemical properties were investigated in a three-electrode cell by using an electrochemical station (Model-660D, CH instruments). The catalyst coated ITO glass, a Pt foil, and an $\mathrm{Ag} / \mathrm{AgCl}$ electrode and $0.5 \mathrm{M} \mathrm{Na}_{2} \mathrm{SO}_{4}$ aqueous solution were used as the working electrode, the counter-electrode, the reference electrode, and the electrolyte, respectively. A $300 \mathrm{~W}$ Xe arc lamp equipped with a $400 \mathrm{~nm}$ cutoff filter was utilized as a light source. EIS were recorded at an applied potential of $0.2 \mathrm{~V}$ versus $\mathrm{Ag} / \mathrm{AgCl}$ 
over the frequency range of $1 \mathrm{MHz} \sim 0.1 \mathrm{~Hz}$. The photocurrent with on/off cycles was measured at an applied potential of $1.0 \mathrm{~V}$ versus $\mathrm{Ag} / \mathrm{AgCl}$. Mott-Schottky plots were obtained under direct current potential polarization at the frequency of $1000 \mathrm{~Hz}$. The potential ranged from -0.6 to $1.0 \mathrm{~V}$ ( $\mathrm{vs} \mathrm{Ag} / \mathrm{AgCl})$.

\section{Results and discussion}

\subsection{Atomic Carbon Chains Linked Polymeric CN}

Generally, the key step for the generation of $\mathrm{CN}$ is the deamination process between amino groups of the precursors and intermediates. ${ }^{[26]}$ Thus, these amino groups also possess the potential to react with $\mathrm{NH}_{2}$ group or $\mathrm{COOH}$ group by releasing $\mathrm{NH}_{3}$ and $\mathrm{H}_{2} \mathrm{O}$ at some specified conditions, resulting in the formation of $\mathrm{C}-\mathrm{N}$ bonds. Inspired by this point, the glycine molecules, with terminated $\mathrm{COOH}$ and $\mathrm{NH}_{2}$ groups and short length, are selected as the potential linker to bridge the in-plane defects of layered CN as illustrated in Scheme 1. The carbon chains linked $\mathrm{CN}$ was prepared by a direct thermal polymerization of melamine and glycine precursor and the obtained samples were denoted as CN-xGly (x means the amount of glycine). We use the glycine as a representative molecule to explore the underlying structure and performance of the products derived from melamine and glycine.

In situ FT-IR was conducted under Ar atmosphere to visualize the incorporating process of carbon chains, in which the detected signal at $100{ }^{\circ} \mathrm{C}$ was used as the background (Figure 1a). The direct polymerization of melamine and glycine is quite a complicated process, wherein abundant old bonds break and new bonds form. To avoid the appearance of various FT-IR signals caused by the transformation of rich bonds and study the potential inserting process of small molecules in the framework of $\mathrm{CN}$ clearly, we loaded glycine molecules on stable pristine $\mathrm{CN}$ and employed this composite as the model for in situ FT-IR observation. The calcination-time dependent infrared spectra (Figure 1a) present three distinct change regions. The signals in regions I and II increased gradually between $150 \sim 250{ }^{\circ} \mathrm{C}$, whereas those in region III declined. When

further increased the temperature up to $450{ }^{\circ} \mathrm{C}$, all typical signals keep unchanged, 
indicating that glycine and $\mathrm{CN}$ could react entirely at approximately $250{ }^{\circ} \mathrm{C}$ and the established structure is steady even at high temperature. Here, we analyze the spectrum at $450{ }^{\circ} \mathrm{C}$ in detail to approach the established structure (Figure 1b). The decreased absorbance at $3450 \mathrm{~cm}^{-1}$ can be ascribed to the consumption of $\mathrm{NH}_{2}$ group, along with the newly appeared NH $\left(3180 \mathrm{~cm}^{-1}\right), \mathrm{C}-\mathrm{N}\left(1038 \mathrm{~cm}^{-1}\right)$ species, ${ }^{[27-29]}$ suggesting that the suspended $\mathrm{NH}_{2}$ groups adjacent to the tri-s-triazine units involved in the reaction with glycine and subsequently generated the $\mathrm{C}-\mathrm{NH}$ motif by releasing $\mathrm{NH}_{3}$ molecules (marked with gray circle in Figure 1c). The new bands at $1119 \mathrm{~cm}^{-1}(\mathrm{C}-\mathrm{N})$ and 1656 $\mathrm{cm}^{-1}(\mathrm{C}=\mathrm{O})$ imply the generation of $\mathrm{N}-\mathrm{C}=\mathrm{O}$ species (marked with cyan circle in Figure 1c), ${ }^{[4]}$ which stemmed from the dehydration reaction between the $(\mathrm{C} 2)-\mathrm{NH}$ motif of $\mathrm{CN}$ and the terminal $\mathrm{COOH}$ groups of glycine molecules. According to the newly formed $\mathrm{C}-\mathrm{N}$ and $\mathrm{N}-\mathrm{C}=\mathrm{O}$ structural motifs, the structure schemed in Figure 1c is proposed. Meanwhile, another similar moiety $\mathrm{NH}-\mathrm{C}=\mathrm{O}$ (marked with red circle in Figure 1d), obtained from the dehydration reaction between the $\mathrm{NH}_{2}$ over $\mathrm{CN}$ and the $\mathrm{COOH}$ group of glycine molecule, also formed as identified by the bands located at $1338 \mathrm{~cm}^{-1}$ and $1531 \mathrm{~cm}^{-1}$ (reason for the different band position of $\mathrm{N}-\mathrm{C}=\mathrm{O}$ and $\mathrm{NH}-\mathrm{C}=\mathrm{O}$ motifs provided in SI-Discussion-1). For comparison, the different signals of pure $\mathrm{CN}$ and the glycine loaded $\mathrm{Al}_{2} \mathrm{O}_{3}$ from that of glycine loaded $\mathrm{CN}$ under same conditions further confirm that the glycine molecule chains indeed inserted into the framework of $\mathrm{CN}$ in the demonstrated way (Figure S1 and SI-Discussion-2). In brief, the analyses above identify that the carbon chains are indeed inserted into the defect of $\mathrm{CN}$ (Scheme 1b) and exist as two forms as illustrated in Figure 1c,d.

The chemical structure and local electronic configuration of the $\mathrm{CN}$ with and without carbon chains were further examined by the synchrotron-based X-ray absorption spectroscopy (XAS) and X-ray photoelectron spectroscopy (XPS). The carbon K-edge XA spectra (Figure 1e) present characteristic resonances of samples including $\pi^{*}$ transitions in $\mathrm{C}=\mathrm{C}$ at $284.7 \mathrm{eV}, \mathrm{N}=\mathrm{C}-\mathrm{N}$ at $287.5 \mathrm{eV}$ and $\sigma^{*}$ transitions in $\mathrm{C}-\mathrm{C}$ between 290 and $298 \mathrm{eV} \cdot{ }^{[30]}$ The intensity ratio of $\sigma^{*}$ transition (in C-C) to $\pi^{*}$ transitions (in $\mathrm{N}=\mathrm{C}-\mathrm{N}$ ) of $\mathrm{CN}-10 \mathrm{Gly}$ was higher than that of pristine $\mathrm{CN}$, which could be ascribed to 
the additional C-C species from the doped carbon chains. (Note: $\mathrm{N}=\mathrm{C}-\mathrm{N}$ is the inherent motif in $\mathrm{CN}$, thus it can be regarded as the reference for the qualitative study of the induced foreign units.) In the nitrogen K-edge region (Figure 1f), typical $\pi^{*}$ resonances at 399.6 and $402.3 \mathrm{eV}$ are observed, corresponding to aromatic nitrogen atoms of heterocyclic rings $\left(\pi^{*} \mathrm{C}=\mathrm{N}-\mathrm{C}\right)$ and $\mathrm{sp}^{3} \mathrm{~N}-\mathrm{C}$ bridging among tri-s-triazine moieties, respectively. ${ }^{[30,31]}$ The $1 \mathrm{~s} \rightarrow \sigma^{*} \mathrm{~N}-\mathrm{C}$ transition is located in the range of 404 to 412 $\mathrm{eV} .{ }^{[31,32]}$ Taking the intensity of intrinsic $\pi^{*} \mathrm{C}=\mathrm{N}-\mathrm{C}$ as reference, the $\mathrm{CN}-10 \mathrm{Gly}$ sample delivered higher intensity of $\sigma^{*} \mathrm{~N}-\mathrm{C}$ relative to that of $\mathrm{CN}$, suggesting a slightly higher content of $\mathrm{C}-\mathrm{N}$ bonds in the carbon chains doped $\mathrm{CN}$. It reveals how the carbon chains link with the matrix of $\mathrm{CN}$, i.e., via covalent bonds between $\mathrm{C}$ and $\mathrm{N}$ as illustrated in Figure 1c,d. The C and N K-edges XA spectra (Figure S2a and Figure S2b) of CN10Gly-(two steps) (synthesis details provided in Supporting Information) show similar results with those of $\mathrm{CN}-10 \mathrm{Gly}$, identifying the rationality of the adopted model, i.e., glycine molecules loaded unmodified CN, used in situ FT-IR examination. The highresolution C $1 s$ XPS spectra (Figure $1 \mathrm{~g}$ ) for pristine $\mathrm{CN}$ can be deconvoluted into two components located at $288.25 \mathrm{eV}$ and $284.78 \mathrm{eV}$, assigned to $\mathrm{N}-\mathrm{C}=\mathrm{N}$ motifs and adventitious hydrocarbons (in the form of $\mathrm{C}-\mathrm{C}$ or $\mathrm{C}=\mathrm{C}$ ), respectively. It was reported that the glycine loaded on graphene oxide showed a close binding energy at $284.6 \mathrm{eV}$, corresponding to the $\mathrm{C}-\mathrm{C} / \mathrm{C}-\mathrm{H}$ species in glycine. ${ }^{[33]}$ Hence, the peak of designed carbon chains could overlap with the adventitious hydrocarbons due to the similar binding energy. Upon introducing the glycine into the feedstock (CN-10Gly), the percentage of $\mathrm{C}-\mathrm{C} / \mathrm{C}=\mathrm{C}$ species to all detected carbon increased from $12.5 \%$ to $21.4 \%$ (Figure 1g and Table S1), clearly suggesting that additional C-C chains contained in the CN-10Gly sample. The slightly increased atomic percentage of $\mathrm{C}$ and $\mathrm{O}$ (derived from XPS) in CN-10Gly relative to that of $\mathrm{CN}$ (Table S2) as well as the appearance of $\mathrm{C}=\mathrm{O}$ species (Figure S3), also discloses the successful incorporation of carbon chains in the $\mathrm{CN}$.

The incorporation of carbon chains in $\mathrm{CN}$ was further verified by the UV-visible spectra, common-used FT-IR and solid-state NMR (Figure S4,5,6; details provided in 
SI-Discussion-3). Apart from the reactive groups, i.e., $\mathrm{COOH}$ and $\mathrm{NH}_{2}$, on the molecule chains, the match of dimension between the inserting molecules and defects of $\mathrm{CN}$ could be another critical factor that determines the incorporation of carbon chains into layers of CN. It's preliminarily identified that the size of inserted carbon chains is comparable to that of defects of $\mathrm{CN}$ according to the XRD analysis (Figure S7a,b and SI-Discussion-4). Based on the above analyses, the carbon chains were successfully planted in the basal plane of $\mathrm{CN}$ and connected the tri-s-triazine units through strong C-N bonds.

\subsection{Carbon Chains-Mediated Photocarriers Separation and Transport}

The modified $\mathrm{CN}$ with carbon chains remained comparable morphology and surface area to that of $\mathrm{CN}$ (Figure S8 and Figure S9), further illustrating that the carbon chains were embedded in the framework of $\mathrm{CN}$ in manner of molecular distribution as demonstrated above. Besides, the doped carbon chains barely affected the band structure (Figure S10) including the band gap (Figure S10a), flat-band potential (Figure S10b) and valence band (Figure S10c).

Usually, carbon materials, such as carbon nanotube, graphene, and even the doped carbon matrix in basal plane of $\mathrm{CN}$, own excellent electrical conductivity. Therefore, we believe that the carbon chains with two $\mathrm{C}$ atoms interconnected with the intrinsic structure of $\mathrm{CN}$ covalently could enable the electron with faster mobility throughout the layers compared with the original layers full of hydrogen bonds. Hence, we focus on the study related to the kinetics of photocarriers in the $\mathrm{CN}$ with and without carbon chains modification. Steady-state/time-resolved photoluminescence (PL) spectroscopy characterizations, as powerful tools to monitor the excitonic processes, i.e., radiative recombination of photogenerated electron-hole pairs in photocatalyst, were carried out for a series of samples. PL intensity represents the combination possibility of lightinduced electron-hole pairs. ${ }^{[34]}$ As shown in Figure 2a, pristine $\mathrm{CN}$ gives a sharp emission peak at $470 \mathrm{~nm}$, but the PL intensity is progressively and substantially suppressed with the increased incorporation amount of carbon chains. This 
phenomenon means greatly enhanced separation and transfer of photocarriers, as profited from promotional effect of the carbon chains. In particular, time-resolved PL spectra of the doped samples (Figure 2b) detected at the emission wavelength, i.e., 470 $\mathrm{nm}$, show the gradual and apparent decrease in fluorescence lifetime (fitted from the decay spectra, Table S3) in comparison with the unmodified CN. The fluorescence decay of the as-prepared samples is entirely in line with their decrease tendency of PL intensities, suggesting faster separation and transfer of electrons and holes in the framework of $\mathrm{CN} .{ }^{[6]}$ It signifies that the more photo-generated carriers captured by reactive substrates under reaction conditions, the more efficient photocatalysis reactions realized for carbon chains linked $\mathrm{CN}$.

We also used photoelectrochemical characterizations to investigate the carriers separation and transfer behavior of as-prepared samples. Nyquist plot of CN-10Gly under the dark condition presents apparently declined semicircles in comparison with that of the pristine $\mathrm{CN}$ (Figure S11), indicative of the more favorable inherent electron transport ability. Upon irradiation with visible light, both of them show decreased semicircles and the carbon chains modified sample still exhibits smaller resistance value compared to the pristine $\mathrm{CN}$ (Figure 2c). Above results suggest a significantly improved electronic conductivity in the non-photoexcited and photoexcited status. Meanwhile, a marked enhancement of photocurrent (inset of Figure 2c) is also observed for $\mathrm{CN}-10 \mathrm{Gly}$ relative to pristine $\mathrm{CN}$, which further illustrates that the mobility of the photo-carriers is promoted remarkably. By combining the results as mentioned earlier, we can reasonably conclude that the embedded carbon chains in the plane of $\mathrm{CN}$ can dramatically promote the separation and transfer of photocarriers with framework of $\mathrm{CN}$.

\subsection{Superior Light-Driven $\mathrm{H}_{2}$ Evolution and $\mathrm{CO}_{2}$ Reduction}

To assess the validity of carbon chains integration strategy over $\mathrm{CN}$ on improving photocatalytic efficiency, photocatalytic $\mathrm{H}_{2}$ evolution was carried out over the assynthesized samples. All the samples modified with various carbon chains (Figure 3a) 
exhibit substantial improvement in photocatalytic $\mathrm{H}_{2}$ evolution in contrast to pristine CN. Particularly, CN-10Gly presents the highest $\mathrm{H}_{2}$ evolution activity, increased by 13.2 times in comparison with that of pristine $\mathrm{CN}$. Our strategy is not only quite simple but also achieves a high activity, i.e., among the top records for the in-plane engineered $\mathrm{CN}$ in $\mathrm{H}_{2}$ evolution (Table $\mathrm{S} 4$ ). This modification strategy also dramatically boosts the photocatalytic $\mathrm{CO}_{2}$ reduction performance because more photoelectrons are available for reactants at the interface between photocatalyst and reaction solution. The optimized sample (CN-10Gly) exhibits a 29.2 times enhancement relative to $\mathrm{CN}$ in $\mathrm{CO}$ generation (Figure $3 b$ ). Both the gaseous products for water splitting and $\mathrm{CO}_{2}$ reduction over the optimized sample increased linearly with the irradiation time (Figure S12), suggesting good stability for $\mathrm{CN}-10 \mathrm{Gly}$. It is further evidenced by the long-term $\mathrm{H}_{2}$ evolution as shown in Figure 3c, only a slight decrease was observed during the 20 hours test. An adverse effect emerged for both $\mathrm{H}_{2}$ evolution (Figure $3 \mathrm{a}$ ) and $\mathrm{CO}$ evolution (Figure 3b) when excessive glycine was introduced. Actually, a similar phenomenon has also been revealed in the relevant studies. ${ }^{[35,36]}$ The negative effect could be ascribed to the fact that introducing too many foreign units may inevitably destruct the conjugated structures of $\mathrm{CN} .{ }^{[35,36]}$ As a result, it could diminish the positive effect of efficient separation of photocarriers, leading to a decline in photocatalytic activity. Interestingly, when maintaining the terminal groups as $\mathrm{COOH}$ and $\mathrm{NH}_{2}$ but increasing the carbon atom number in the doping molecule from two to four, the activity of samples presents a drastic change (Figure S13). When the carbon number is three, the $\mathrm{H}_{2}$ evolution rate, reaching up to $\sim 167 \mu \mathrm{mol} / \mathrm{h}$, is almost twice that of glycine (carbon number is equal to two) modified sample $(90 \mu \mathrm{mol} / \mathrm{h})$ and 24.6 folds higher than pristine $\mathrm{CN}$. However, the activity $(23 \mu \mathrm{mol} / \mathrm{h})$ decreases drastically as the carbon number further increased to four. We preliminarily ascribe this phenomenon to the match degree of size between defects and molecules, that is, the dimension of molecules containing two or three carbon atoms is comparable to that of defects; however, the molecules with four carbon atoms are hard to be embedded into the defects of $\mathrm{CN}$. Further studies are still needed to validate this assumption. 
To further demonstrate the universality of this strategy, various precursors and molecule linkers (Figure S14) have been adopted. All the as-prepared samples (Figure 3d,e) exhibited significant enhancement in $\mathrm{H}_{2}$ evolution. (Note: these molecule chains linkers, i.e., L-cysteine, Allyl thiourea and L-Arginine, possess groups on adjacent C atoms, including $\mathrm{NH}, \mathrm{NH}_{2}$ and $\mathrm{COOH}$ groups, which are apt to react with the residual $\mathrm{NH}_{2}$ or $\mathrm{NH}$ groups in $\mathrm{CN}$.)

\section{Conclusions}

In conclusion, our study demonstrates a facile and efficient strategy to incorporate short carbon chains into the basal plane of $\mathrm{CN}$ while remaining the pristine tri-s-triazine structure. The carbon chains filled the defects in $\mathrm{CN}$ via covalent bonds, which mediates the electron transfer in a relatively smooth transport channel compared to the initial hydrogen bonds. By virtue of such a favorable transport channel of carriers, the carbon chains linked $\mathrm{CN}$ exhibits a dramatically improved photocatalytic water splitting and $\mathrm{CO}_{2}$ reduction performance, achieving a 13.2 and 29.2 times enhancement, respectively, compared to pristine $\mathrm{CN}$. This study highlights that the engineering of the defects in photocatalysts is one of the most effective approaches for improving solar energy conversion and also gives a research direction for the future rational design towards efficient photocatalysts, especially for organic-based ones.

\section{Acknowledgements}

This work received financial support from the World Premier International Research Center Initiative (WPI Initiative) on Materials Nanoarchitectonics (MANA), MEXT, Japan.

\section{Appendix A. Supplementary data}

Supplementary data associated with this article can be found in the online version, at http://dx.doi.org/10.1016/j.apcatb.xxxxxxx.

\section{References}


[1] S.N. Habisreutinger, L. Schmidt-Mende, J.K. Stolarczyk, Photocatalytic reduction of $\mathrm{CO}_{2}$ on $\mathrm{TiO}_{2}$ and other semiconductors, Angew Chem Int Ed 52 (2013) 7372-7408.

[2] X. Wang, K. Maeda, A. Thomas, K. Takanabe, G. Xin, J.M. Carlsson, K. Domen, M. Antonietti, A metal-free polymeric photocatalyst for hydrogen production from water under visible light, Nat. Mater., 8 (2009) 76-80.

[3] G. Zhang, G. Li, T. Heil, S. Zafeiratos, A. Savateev, X. Wang, M. Antonietti, Tailoring Grain Boundary Chemistry of Polymeric Carbon Nitride for Enhanced Solar $\mathrm{H}_{2}$ Production and $\mathrm{CO}_{2}$ Reduction, Angew. Chem. Int. Ed., 58 (2019) 3433-3437.

[4] G. Zhao, H. Pang, G. Liu, P. Li, H. Liu, H. Zhang, L. Shi, J. Ye, Coporphyrin/carbon nitride hybrids for improved photocatalytic $\mathrm{CO}_{2}$ reduction under visible light, Appl. Catal., B, 200 (2017) 141-149.

[5] P. Yang, H. Zhuzhang, R. Wang, W. Lin, X. Wang, Carbon Vacancies in a Melon Polymeric Matrix Promote Photocatalytic Carbon Dioxide Conversion, Angew. Chem. Int. Ed. Engl., 58 (2019) 1134-1137.

[6] Y. Li, S. Ouyang, H. Xu, X. Wang, Y. Bi, Y.Zhang, J. Ye, Constructing Solid-GasInterfacial Fenton Reaction over Alkalinized- $\mathrm{C}_{3} \mathrm{~N}_{4}$ Photocatalyst To Achieve Apparent Quantum Yield of 49\% at 420 nm, J. Am. Chem. Soc., 138 (2016) 13289-13297.

[7] N. Tian, K. Xiao, Y. Zhang, X. Lu, L. Ye, P. Gao, T. Ma, H. Huang, Reactive sites rich porous tubular yolk-shell $\mathrm{g}-\mathrm{C}_{3} \mathrm{~N}_{4}$ via precursor recrystallization mediated microstructure engineering for photoreduction, Appl. Catal., B, 253 (2019) 196-205.

[8] J. Cai, J. Huang, S. Wang, J. Iocozzia, Z. Sun, J. Sun, Y. Yang, Y. Lai, Z. Lin, Crafting Mussel-Inspired Metal Nanoparticle-Decorated Ultrathin Graphitic Carbon Nitride for the Degradation of Chemical Pollutants and Production of Chemical Resources, Adv. Mater., 31 (2019) e1806314.

[9] G. Liao, Y. Gong, L. Zhang, H. Gao, G.-J. Yang, B. Fang, Semiconductor polymeric graphitic carbon nitride photocatalysts: the "holy grail" for the photocatalytic hydrogen 
evolution reaction under visible light, Energy Environ. Sci., (2019), DOI: 10.1039/c9ee00717b.

[10] W. Cui, J. Li, Y. Sun, H. Wang, G. Jiang, S.C. Lee, F. Dong, Enhancing ROS generation and suppressing toxic intermediate production in photocatalytic NO oxidation on $\mathrm{O} / \mathrm{Ba}$ co-functionalized amorphous carbon nitride, Appl. Catal., B, 237 (2018) 938-946.

[11] J. Ji, J. Wen, Y. Shen, Y. Lv, Y. Chen, S. Liu, H. Ma, Y. Zhang, Simultaneous Noncovalent Modification and Exfoliation of 2D Carbon Nitride for Enhanced Electrochemiluminescent Biosensing, J. Am. Chem. Soc., 139 (2017) 11698-11701.

[12] J. Li, D. Wu, J. Iocozzia, H. Du, X. Liu, Y. Yuan, W. Zhou, Z. Li, Z. Xue, Z. Lin, Achieving efficient incorporation of П-electrons into graphitic carbon nitride for markedly improved hydrogen generation, Angew. Chem., 58 (2019) 1985-1989.

[13] Z. Teng, N. Yang, H. Lv, S. Wang, M. Hu, C. Wang, D. Wang, G. Wang, EdgeFunctionalized g-C3N4 Nanosheets as a Highly Efficient Metal-free Photocatalyst for Safe Drinking Water, Chem, 5 (2019) 664-680.

[14] H. Yu, L. Shang, T. Bian, R. Shi, G.I. Waterhouse, Y. Zhao, C. Zhou, L.Z. Wu, C.H. Tung, T. Zhang, Nitrogen-Doped Porous Carbon Nanosheets Templated from g$\mathrm{C}_{3} \mathrm{~N}_{4}$ as Metal-Free Electrocatalysts for Efficient Oxygen Reduction Reaction, Adv. Mater., 28 (2016) 5080-5086.

[15] Z. Chen, E. Vorobyeva, S. Mitchell, E. Fako, M.A. Ortuño, N. López, S.M. Collins, P.A. Midgley, S. Richard, G. Vilé, J. Pérez-Ramírez, A heterogeneous single-atom palladium catalyst surpassing homogeneous systems for Suzuki coupling, Nat. Nanotechnol., 13 (2018) 702-707.

[16] P. Yang, L. Wang, H. Zhuzhang, R. Wang, M.-M. Titirici, X. Wang, Photocarving nitrogen vacancies in a polymeric carbon nitride for metal-free oxygen synthesis, Appl. Catal., B, 256 (2019). 
[17] Y. Li, H. Xu, S. Ouyang, D. Lu, X. Wang, D. Wang, J. Ye, In situ surface alkalinized g- $\mathrm{C}_{3} \mathrm{~N}_{4}$ toward enhancement of photocatalytic $\mathrm{H}_{2}$ evolution under visiblelight irradiation, J. Mater. Chem. A, 4 (2016) 2943-2950.

[18] Y. Zheng, J. Liu, J. Liang, M. Jaroniec, S.Z. Qiao, Graphitic carbon nitride materials: controllable synthesis and applications in fuel cells and photocatalysis, Energy Environ. Sci., 5 (2012).

[19] P. Qiu, C. Xu, N. Zhou, H. Chen, F. Jiang, Metal-free black phosphorus nanosheets-decorated graphitic carbon nitride nanosheets with C-P bonds for excellent photocatalytic nitrogen fixation, Appl. Catal., B, 221 (2018) 27-35.

[20] Y. Kang, Y. Yang, L.C. Yin, X. Kang, L. Wang, G. Liu, H.M. Cheng, Selective Breaking of Hydrogen Bonds of Layered Carbon Nitride for Visible Light Photocatalysis, Adv. Mater., 28 (2016) 6471-6477.

[21] B.V. Lotsch, M. Döblinger, J. Sehnert, L. Seyfarth, J. Senker, O. Oeckler, W. Schnick, Unmasking Melon by a Complementary Approach Employing Electron Diffraction, Solid-State NMR Spectroscopy, and Theoretical Calculations-Structural Characterization of a Carbon Nitride Polymer, Chem. - Eur. J., 13 (2007) 4969-4980.

[22] L. Lin, H. Ou, Y. Zhang, X. Wang, Tri-s-triazine-Based Crystalline Graphitic Carbon Nitrides for Highly Efficient Hydrogen Evolution Photocatalysis, ACS Catal., 6 (2016) 3921-3931.

[23] W. Che, W. Cheng, T. Yao, F. Tang, W. Liu, H. Su, Y. Huang, Q. Liu, J. Liu, F. Hu, Z. Pan, Z. Sun, S. Wei, Fast Photoelectron Transfer in (Cring)- $\mathrm{C}_{3} \mathrm{~N}_{4}$ Plane Heterostructural Nanosheets for Overall Water Splitting, J. Am. Chem. Soc., 139 (2017) 3021-3026.

[24] Y. Yu, W. Yan, X. Wang, P. Li, W. Gao, H. Zou, S. Wu, K. Ding, Surface Engineering for Extremely Enhanced Charge Separation and Photocatalytic Hydrogen Evolution on g- $\mathrm{C}_{3} \mathrm{~N}_{4}$, Adv. Mater., 30 (2018) 1705060. 
[25] Y. Wang, X. Liu, J. Liu, B. Han, X. Hu, F. Yang, Z. Xu, Y. Li, S. Jia, Z. Li, Y. Zhao, Carbon Quantum Dot Implanted Graphite Carbon Nitride Nanotubes: Excellent Charge Separation and Enhanced Photocatalytic Hydrogen Evolution, Angew. Chem. Int. Ed., 57 (2018) 5765-5771.

[26] A. Thomas, A. Fischer, F. Goettmann, M. Antonietti, J.-O. Müller, R. Schlögl, J.M. Carlsson, Graphitic carbon nitride materials: variation of structure and morphology and their use as metal-free catalysts, J. Mater. Chem., 18 (2008) 4893-4908.

[27] G. Zhao, Y. Sun, W. Zhou, X. Wang, K. Chang, G. Liu, H. Liu, T. Kako, J. Ye, Superior Photocatalytic $\mathrm{H}_{2}$ Production with Cocatalytic Co/Ni Species Anchored on Sulfide Semiconductor, Adv. Mater., 29 (2017).

[28] H. Yu, R. Shi, Y. Zhao, T. Bian, Y. Zhao, C. Zhou, G.I.N. Waterhouse, L.Z. Wu, C.H. Tung, T. Zhang, Alkali-Assisted Synthesis of Nitrogen Deficient Graphitic Carbon Nitride with Tunable Band Structures for Efficient Visible-Light-Driven Hydrogen Evolution, Adv. Mater., 29 (2017) 1605148-1605154.

[29] P. Niu, L. Zhang, G. Liu, H.-M. Cheng, Graphene-Like Carbon Nitride Nanosheets for Improved Photocatalytic Activities, Adv. Funct. Mater., 22 (2012) 4763-4770.

[30] Y. Zheng, Y. Jiao, Y. Zhu, L.H. Li, Y. Han, Y. Chen, A. Du, M. Jaroniec, S.Z. Qiao, Hydrogen evolution by a metal-free electrocatalyst, Nat. Commun., 5 (2014) 3783.

[31] N. Meng, J. Ren, Y. Liu, Y. Huang, T. Petit, B. Zhang, Engineering oxygencontaining and amino groups into two-dimensional atomically-thin porous polymeric carbon nitrogen for enhanced photocatalytic hydrogen production, Energy Environ. Sci., $11(2018)$ 566-571.

[32] H.B. Yang, J. Miao, S.F. Hung, J. Chen, H.B. Tao, X. Wang, L. Zhang, R. Chen, J. Gao, H.M. Chen, L. Dai, B. Liu, Identification of catalytic sites for oxygen reduction and oxygen evolution in $\mathrm{N}$-doped graphene materials: Development of highly efficient metal-free bifunctional electrocatalyst, Sci. Adv., 2 (2016) e1501122. 
[33] K. Pytlakowska, V. Kozik, M. Matussek, M. Pilch, B. Hachuła, K. Kocot, Glycine modified graphene oxide as a novel sorbent for preconcentration of chromium, copper, and zinc ions from water samples prior to energy dispersive X-ray fluorescence spectrometric determination, RSC Adv., 6 (2016) 42836-42844.

[34] D. Zheng, X.N. Cao, X. Wang, Precise Formation of a Hollow Carbon Nitride Structure with a Janus Surface To Promote Water Splitting by Photoredox Catalysis, Angew. Chem. Int. Ed., 55 (2016) 11512-11516.

[35] J. Zhang, G. Zhang, X. Chen, S. Lin, L. Mohlmann, G. Dolega, G. Lipner, M. Antonietti, S. Blechert, X. Wang, Co-monomer control of carbon nitride semiconductors to optimize hydrogen evolution with visible light, Angew. Chem. Int. Ed., 51 (2012) 3183-3187.

[36] J. Qin, S. Wang, H. Ren, Y. Hou, X. Wang, Photocatalytic reduction of $\mathrm{CO}_{2}$ by graphitic carbon nitride polymers derived from urea and barbituric acid, Appl. Catal., B, 179 (2015) 1-8. 


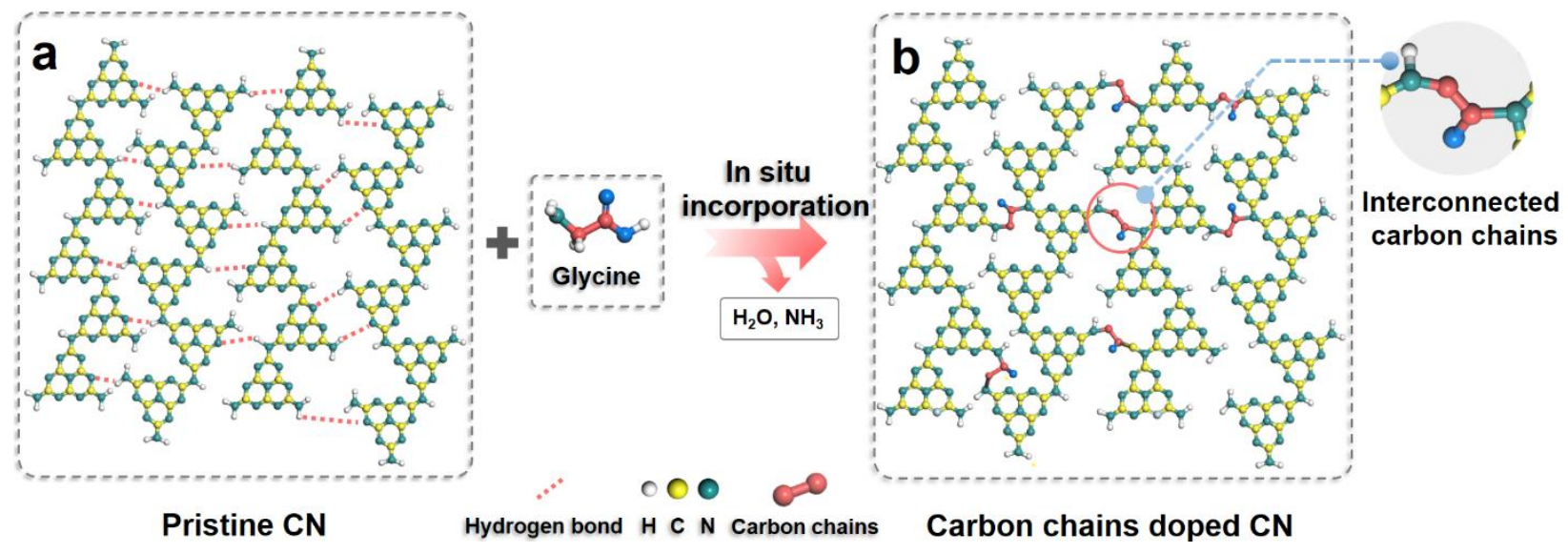

Scheme 1. Structure of $\mathrm{CN}$ before a) and after b) doping with carbon chains. In the doping process, the glycine molecules were evolved into the reaction with terminal groups, i.e., $\mathrm{NH}_{2}$ and $\mathrm{NH}$, in $\mathrm{CN}$, and thus the carbon chains were interconnected with the tri-s-triazine units via $\mathrm{C}-\mathrm{N}$ bonds. 

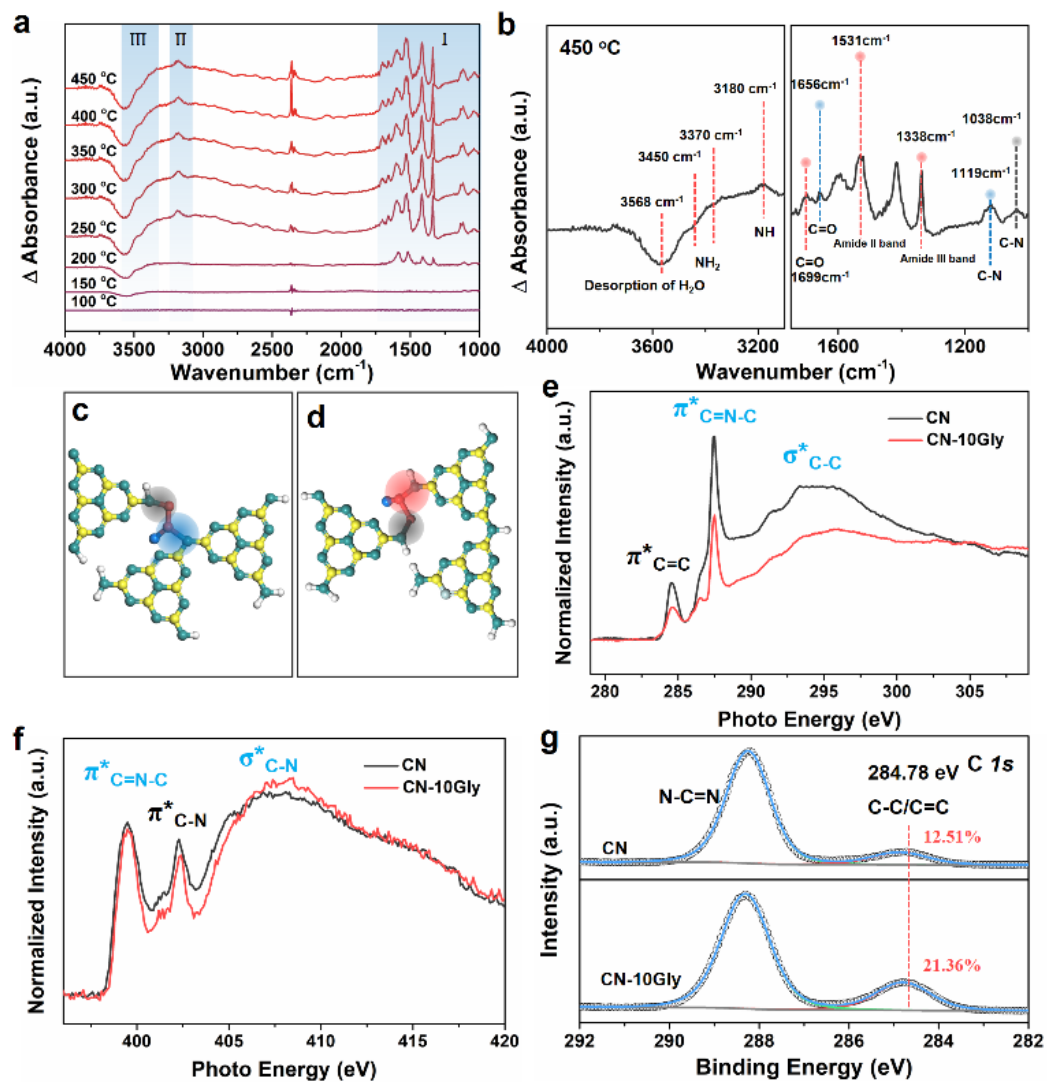

Figure 1. Structure identification of the carbon chains incorporated CN. a) In situ FT-IR spectra of glycine loaded CN measured at different temperature. b) The in situ FT-IR spectrum at $450{ }^{\circ} \mathrm{C}$. c,d) The illustration of two possible kinds of structure in the carbon chains doped CN. e,f) Carbon and nitrogen K-edge XA spectra of $\mathrm{CN}$ and $\mathrm{CN}$ 10Gly, respectively. g) The C $1 s$ spectra of CN and CN-10Gly. 

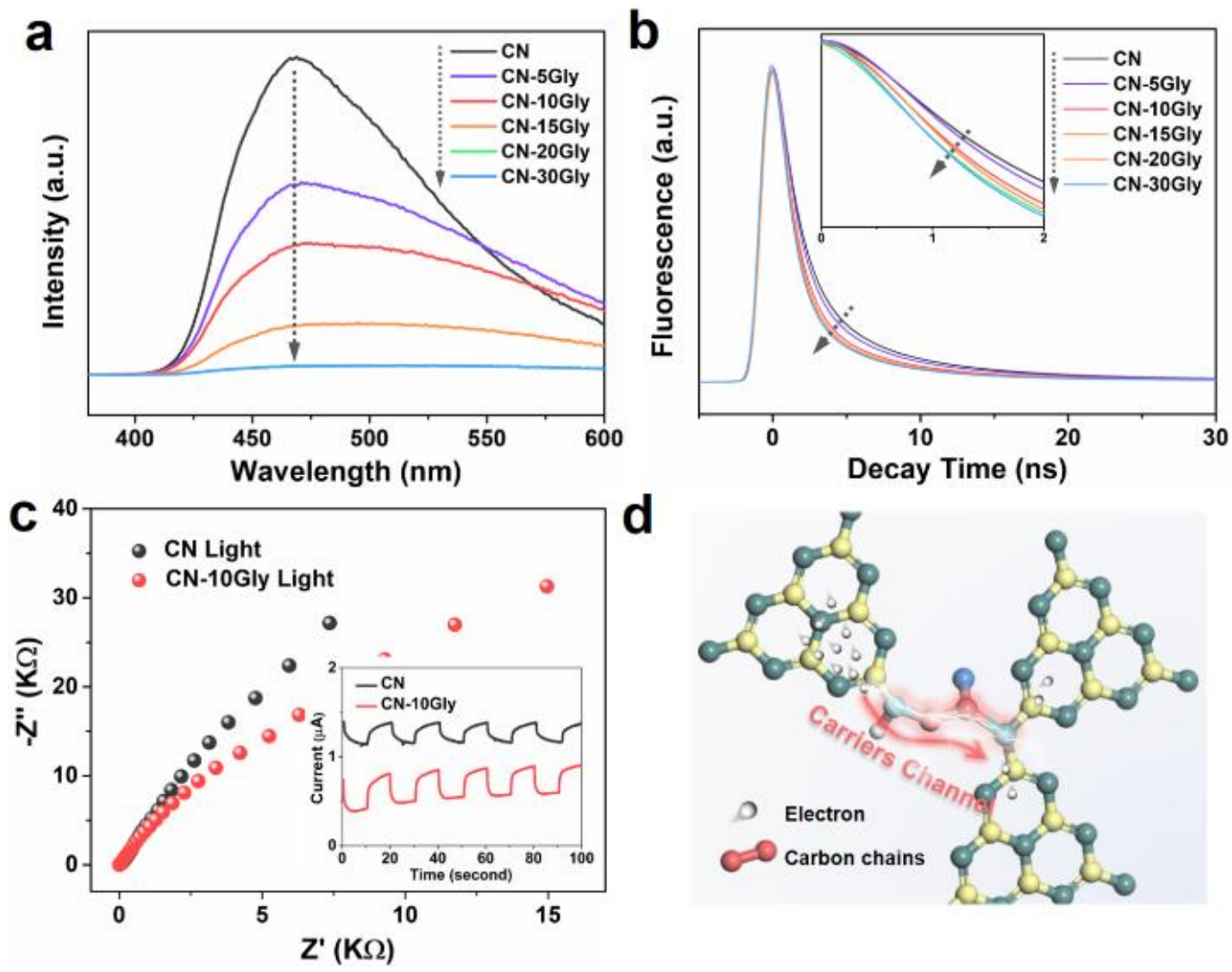

Figure 2. Characterization of the photocarriers kinetics of the as-prepared samples. a,b) Steady-state and time-resolved photoluminescence spectroscopy of the samples, respectively. c) Electrochemical impedance spectroscopy (EIS) Nyquist plots under irradiation condition. Inset: Periodic on/off photocurrent response under visiblelight irradiation. d) Schematic diagram of carriers channel role of bridged carbon chains. 

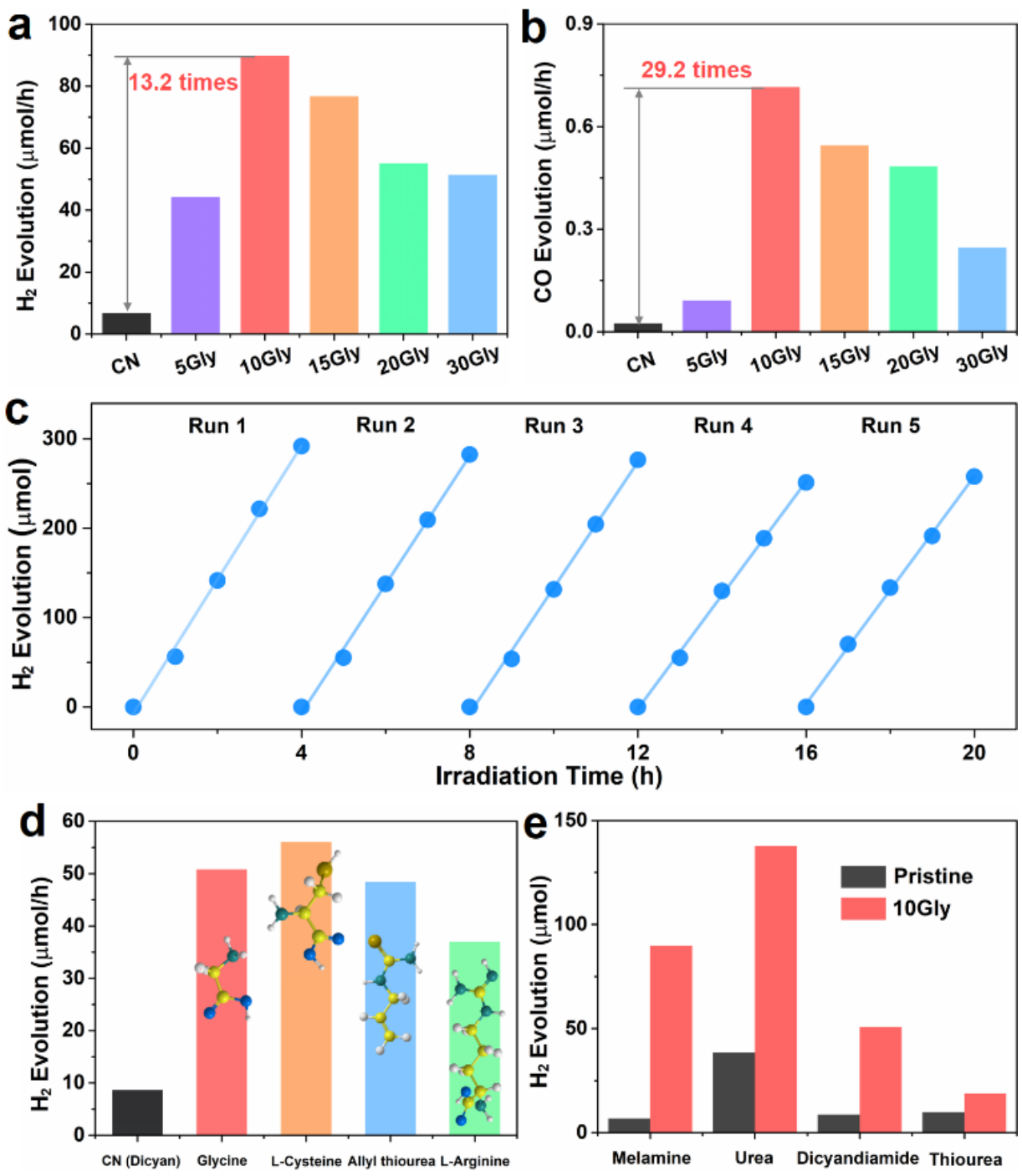

Figure 3. Photocatalytic activity evaluation of the as-prepared samples. a,b) Comparison of the photocatalytic $\mathrm{H}_{2}$ evolution and $\mathrm{CO}_{2}$ reduction rate of the samples with different amount of glycine, respectively. c) Cycle test of $\mathrm{H}_{2}$ evolution over $\mathrm{CN}$ 10Gly. d) Comparison of the photocatalytic $\mathrm{H}_{2}$ evolution activity of samples obtained from dicyandiamide and different kinds of small molecules. e) Comparison of the photocatalytic $\mathrm{H}_{2}$ evolution activity of samples derived from glycine and different precursors. 